\title{
Attention deficit hyperactivity disorder symptom self-report among medical students in Eldoret, Kenya
}

\author{
L Atwoli, P Owiti, G Manguro, D Ndambuki
}

Moi University School of Medicine, Eldoret, Kenya

\begin{abstract}
Objective: To determine the prevalence of self-reported attention deficit hyperactivity disorder (ADHD) symptoms among medical students in Eldoret, Kenya. Method: A cross-sectional descriptive study of all medical students who gave consent to participate in the study. Undertaken at Moi University's School of Medicine in Eldoret, Kenya. Comprising two hundred and fifty three (253) undergraduate medical students, with a mean age of 23.7 years (19-42, s.d. 4.1), of whom 51\% were female. Measuring ADHD symptomatology using the Adult ADHD Self-Report Scale (ASRS v1.1). Results: The prevalence rate of self-reported ADHD symptoms using the ASRS screener was $23.7 \%$. This was significantly associated with being in the age-group 17-20 years compared $(\mathrm{p}<0.05)$. The prevalence rate was higher among females (25.6\%) than among males (21.8\%), but this difference was not statistically significant. Preclinical students had a higher prevalence rate of ADHD symptoms (28.7\%) compared to clinical students (19.6\%), but this was also not statistically significant. Using a modification of the ASRS full symptom checklist to approximate a Diagnostic and Statistical Manual of Mental Disorders, 4th edition, text revision (DSM-IV-TR) ADHD diagnosis yielded a 'possible ADHD' prevalence rate of $8.7 \%$. Of these, the inattentive type was the most common (40.9\%). Conclusion: The prevalence rate of self-reported ADHD symptoms among medical students in Eldoret is very high and possibly interferes with the students' social and academic functioning. Further studies are suggested to generate information on the real $\mathrm{ADHD}$ prevalence in the general population and in special populations such as schools and colleges.
\end{abstract}

Key words: Attention Deficit Hyperactivity Disorder (ADHD); Medical Students; Prevalence; Self-Report

Received: $25-10-2010$

Accepted: $22-11-2010$

doi: http://dx.doi.org/10.4314/ajpsy.v14i4.5

\section{Introduction}

Studies on ADHD prevalence have focussed mainly on children, and only recently has adult ADHD become an area of interest in research. Indeed, the Diagnostic and Statistical Manual of Mental Disorders, 4th edition, text revision, (DSM-IV-TR) criteria for ADHD seems to focus on childhood symptoms, and implies that a significant proportion of patients are symptom-free by early to middle adulthood. ${ }^{1}$ In population based studies, the prevalence rate of $\mathrm{ADHD}$ among children and adolescents has ranged from $1-7.3 \%$ with wide variations reported in many studies. ${ }^{2-5}$ Some studies have estimated the worldwide pooled prevalence of ADHD to be 5.29\%. ${ }^{2,6}$

\section{Correspondence}

Dr Lukoye Atwoli

Department of Mental health, Moi University School of Medicine,

PO Box 1493 Eldoret 30100

email: lukoye@gmail.com
Interest in adult $\mathrm{ADHD}$ is a relatively recent development, given that most of the early prevalence studies did not include adults in their samples, ${ }^{5,7,8}$ In many cases, treatment has not been considered useful beyond adolescence. ${ }^{9}$ Despite earlier suggestions that symptoms completely or significantly remit before adulthood, recent work indicates that a significant proportion of adults with ADHD continue to suffer considerable dysfunction due to their symptoms. , $^{4,8-10}$

The estimated adult prevalence rate currently ranges between 3-5\%, but there have been wide variations across studies. ${ }^{4,5,10-12}$ At face value, this lower prevalence of symptoms in adult populations has been taken to mean that most symptoms remit by early adulthood. However, the weight of evidence now suggests that a contributing factor to the perceived decline in prevalence could be the fact that the DSM-IV-TR diagnostic criteria was formulated with children in mind and adults only 'outgrow' the diagnostic criteria, and not the disorder itself. 2,5

ADHD is reported even in institutions of higher learning 
despite the assumption that the symptoms would impair cognitive functioning so severely that most people with $\mathrm{ADHD}$ would not be able to perform well in school.,13,14 Despite predictions of low prevalence rates of $1-3 \%$ in college and university student populations, a study carried out in several universities in three countries (Italy, New Zealand and the United States) found prevalence rates of between $0-8.1 \%{ }^{3}$

Another study found that $7 \%$ of college students suffered significant ADHD symptoms, and suggested a role of these symptoms in causing problems in academic and other important areas of functioning. ${ }^{14}$

Few ADHD studies have been carried out among medical students. In an anonymous survey of medical students in the US, 5.5\% reported having been diagnosed with $\mathrm{ADHD}$, with $72.2 \%$ of these having been diagnosed after the age of 18 years. ${ }^{15}$ Studies from African and developing countries are even more difficult to come by, and this study aimed at increasing the body of knowledge on this condition among medical students in an African population.

\section{Methods}

Site

The study was carried out at the Town Campus of Moi University. The campus is located in Eldoret town, about $320 \mathrm{~km}$ North West of the capital Nairobi, Kenya. The School of Medicine is one of four schools within this campus (Nursing, Dentistry, Public Health and Medicine), and at the time of the study there were 556 registered medical students from the first year to the sixth year.

This study was part of a larger study on ADHD among all the students at the Moi University Town Campus, and this report uses some of the data collected during the larger study. ${ }^{16}$

\section{Participants}

The study aimed at recruiting all the students at the School of medicine (556 students at the time of the study). A total of 253 students were available and accepted to participate in the study. Slightly over half of the participants were female (51.0\%). Table I shows the distribution of the participants by gender and year of study.

\begin{tabular}{|c|c|c|c|c|}
\hline & & Male (\%) & Female (\%) & Total (\%) \\
\hline YEAR OF STUDY & $\begin{array}{l}1 \\
2 \\
3 \\
4 \\
5 \\
6\end{array}$ & $\begin{array}{l}30(52.6) \\
10(40.0) \\
16(48.5) \\
13(44.8) \\
38(67.9) \\
17(32.1)\end{array}$ & $\begin{array}{l}27(47.4) \\
15(60.0) \\
17(51.5) \\
16(55.2) \\
18(32.1) \\
36(67.9)\end{array}$ & $\begin{array}{l}57(22.5) \\
25(9.9) \\
33(13.0) \\
29(11.5) \\
56(22.1) \\
53(20.9)\end{array}$ \\
\hline \multicolumn{2}{|l|}{ TOTAL } & $124(49.0)$ & $129(51.0)$ & $253(100)$ \\
\hline
\end{tabular}

\section{Design}

This was a cross-sectional descriptive survey using the Adult ADHD self-report scale (ASRS v1.1) to determine the prevalence of self-reported ADHD symptoms among medical students in Eldoret, Kenya. The ASRS is a self-administered questionnaire developed by the World Health Organization to be used as a screening tool for adult $\mathrm{ADHD} .{ }^{17}$ It consists of 18 questions derived from the DSM-IV-TR diagnostic criteria for ADHD and is divided into two parts. Part A consists of a 6-question screener that has been validated elsewhere as a sensitive screen for adult ADHD. ${ }^{17,18}$ Studies have even suggested that until populationwide normative data is available to validate the full-symptom checklist, the 6-item screener is to be preferred to the full-item scale for both community surveys and clinical outreach and case-finding initiatives. ${ }^{18}$ The first three questions (Questions 1-3) on the screener (Part A of the questionnaire) require a response in the range of 'sometimes' to 'very often' to be considered positive while the remaining three (Questions 4-6) require an 'often' or 'very often' response. Four positive responses in part A constitute a positive screen for adult ADHD.

Part B consists of the remaining items on the DSM-IV-TR $\mathrm{ADHD}$ diagnostic criteria and is not required for purposes of screening for adult ADHD. However, in this study, an attempt was made to come up with an approximation of the DSM-IV-TR diagnostic criteria for ADHD using the full ASRS symptom checklist. A modification was introduced requiring endorsement of each item as 'often' or 'very often' for a positive score. DSM-IVTR diagnostic criterion A for ADHD was then used to screen for possible $\mathrm{ADHD}$ in the participants. ${ }^{1}$ To meet the criteria for possible ADHD, at least 6 of either the inattentive symptoms (Questions 1-4 and 7-1 1 on the ASRS checklist) or the hyperactivity/impulsivity symptoms (Questions 5, 6 and 12-18 on the ASRS checklist) had to be positive on the full ASRS symptom checklist. Since information on functional impairment and other possible causes of the symptoms was not collected in the questionnaire, the resultant 'possible $\mathrm{ADHD}$ ' diagnosis remained an approximation and all those who screened positive were advised to seek further assistance from a mental health specialist.

A brief questionnaire for collection of socio-demographic data was also used. Data collected included year of birth, gender and year of study.

\section{Procedure}

The study was conducted during the period March to October, 2009. The study protocol was subjected to ethics review by the Institutional Research and Ethics Committee (IREC) of Moi University and Moi Teaching and Referral Hospital.

Questionnaires were distributed according to the known number of students in each class with the assistance of class representatives prior to the start of whole-class activities and collected at the end of the class. Each questionnaire had a detachable section with a unique number linked to the full questionnaire and known only to each individual participant for the purposes of feedback.

\section{Data storage and analysis}

Collected data was entered into a Microsoft Access database and analysed using SPSS version 12.0. Descriptive statistics were used to compute means and standard deviations for numerical variables as well as frequencies for nominal and ordinal variables. The Chi square test statistic $\left(\chi^{2}\right)$ was used to test the significance of association between various factors and a positive ASRS screen. A value of $\mathrm{p}<0.05$ was considered statistically significant.

\section{Feedback}

Every participant received feedback on the screen result and its implication, and those with positive screens were advised to seek a consultation with a mental health specialist. The feedback 
report was only linked to the unique number on each questionnaire, and the results were sealed and delivered through the respective class representatives.

\section{Results}

\section{Study Participants}

The study involved 253 participants with a mean age of 23.7 years (19-42, s.d. 4.1). Twenty eight (11.1\%) of the participants were aged 17-20 years, 127 (50.8\%) were aged 21-24 years and 98 (38.1\%) were 25 years and older. In this study, $51.0 \%$ of the participants were female and $22.5 \%$ were in their first year of study. Table I above shows the distribution of the participants by gender and year of study.

Only one participant indicated that he had been earlier diagnosed with $\mathrm{ADHD}$ and was on treatment at the time of the study.

\section{Self-reported ADHD symptoms}

In this study, the prevalence of self-reported ADHD symptoms using the ASRS screener was 23.7\%. Table II shows the distribution of self-reported ADHD symptoms with various variables. The only variable showing significant association with ADHD symptoms was younger age-group.

\section{Table II: Variation of ADHD with socio-demographic factors}

\begin{tabular}{|l|l|l|l|}
\hline Variable (N) & ADHD \% & $X^{2}$ & $p$ \\
\hline Age-group & & & \\
$17-20(28)$ & 39.3 & 9.606 & $0.008^{*}$ \\
$21-24(127)$ & 27.6 & & \\
25 and older (98) & 14.3 & & \\
Gender & & 0.507 & 0.287 \\
Female (129) & 25.6 & & \\
Male (124) & 21.8 & & \\
Year of study & 28.7 & 2.890 & 0.061 \\
Pre-clinical (115) & 19.6 & & \\
Clinical (138) & & \\
\hline *Statistically significant &
\end{tabular}

Using the modification described in the methods section above (full ASRS symptom checklist) to approximate a DSM IV TR diagnosis, 22 (8.7\%) met the criteria for possible ADHD. A larger proportion of those meeting these criteria had the inattentive type of symptoms. Table III shows the distribution of symptoms by type of $\mathrm{ADHD}$.

\begin{tabular}{|l|l|}
\hline \multicolumn{2}{|l|}{ Table III: Types of ADHD } \\
\hline TYPE & FREQUENCY (\%) \\
\hline Inattentive & $9(40.9)$ \\
Hyperactive/Impulsive & $7(31.8)$ \\
Combined & $6(27.3)$ \\
\hline TOTAL & $22(100)$ \\
\hline
\end{tabular}

\section{Discussion}

There is a limited body of knowledge on adult ADHD in lowincome countries. A literature search yielded little data on ADHD among medical students from these countries. In Kenya, as in many parts of Africa, joining medical school is often difficult and only the best candidates in high school are selected. Selected students then go through a rigorous program lasting five to six years, and many drop out due to the heavy demands on their academic and social life. Due to the problems with attention and concentration associated with $\mathrm{ADHD}$, it is often assumed that its prevalence would be very low among medical students and others pursuing similarly demanding courses.

This study found a self-reported ADHD symptom prevalence rate of $23.7 \%$ among the medical students using the $\mathrm{WHO}$ validated ASRS v1.1 screener. ${ }^{17}$ This rate is much higher than that reported even in the general population in studies in the developed world. 4,5,10-12,14,15 This finding raises two possibilities. One of the possibilities is that the instrument, which has had limited prior application to this population, may be inaccurate and needs proper validation in this setting.

This view is supported by previous work that has suggested that the wide variations in the prevalence of $\mathrm{ADHD}$ are due to different methodologies used in the various studies as opposed to any cultural or geographical differences.5,6

Alternatively, the rates of ADHD in this setting may actually be much higher than those in the developed countries, and the real problem might be under-recognition and under-treatment. The fact that only one of the respondents had been diagnosed and was on treatment for ADHD suggests that the second possibility is closer to the reality than any weaknesses inherent in the instrument itself.

Proceeding on the assumption that the rates found in this study reflect a truly high prevalence rate of $\mathrm{ADHD}$ among medical students would mean that the prevalence in the general population in this setting is even higher. Studies elsewhere have indeed reported higher prevalence rates in the general population than among university and college students. 3,13,14

In this study, the researchers also applied an approximation of the DSM IV ADHD diagnosis using a stricter though untested modification of the ASRS full symptom check list as described in the Methods section above. This yielded a 'possible ADHD' prevalence rate of $8.7 \%$. This rate is still significantly higher than the rates reported in most other studies in adult population..$^{1-5}$ However, it is comparable to that found in a study conducted among university students in Italy, New Zealand and the United States that found rates up to $8.1 \%{ }^{3}$

Another study in the same setting but involving a combination of medical and other health science students found an $\mathrm{ADHD}$ self-reported symptom prevalence rate of $9.2 \%$ using the same criteria. ${ }^{16}$ Though the magnitude of the difference in the prevalence rates is modest, it seems to provide further evidence that $\mathrm{ADHD}$ would interfere with academic and occupational progression as suggested by previous work. 1,3,13,14

This study also suggests an age-dependent decline in the symptoms of $\mathrm{ADHD}$, with the younger age groups showing higher symptom prevalence rates than the older students, consistent with the trend reported elsewhere. ${ }^{8}$ A trend that mirrored this finding was the higher prevalence of $\mathrm{ADHD}$ symptoms among pre-clinical students compared to clinical students, although this difference did not reach statistical significance. 
However, the age-related differences in symptom distribution could also be explained by the fact that due to the rigorous demands of medical school, students with more troubling symptoms may have either dropped out or been discontinued due to academic or disciplinary difficulties. This line of investigation was however not pursued in this study.

Although females had a higher prevalence rate of ADHD symptoms in this study, the gender difference was not statistically significant, a finding similar to what has been reported in studies in Western populations. ${ }^{2}$ It has also been reported that among children $\mathrm{ADHD}$ affects more boys than girls, but these differences are eroded as they grow older. ${ }^{4}$

The finding that only one participant in this study had been diagnosed and was on treatment for ADHD highlights the aforementioned possibility that $\mathrm{ADHD}$ is inadequately diagnosed and treated in this population. This situation is the same in almost all settings where adult ADHD has been studied, and where people with ADHD get medical attention, it is often not for the ADHD but for the emotional, behavioural and other co-morbidities they present with, including antisocial personality, mood, anxiety, and substance use disorders. $4,5,7,19$

Using the full ASRS modification to approximate a DSM IV TR ADHD diagnosis as described in the methods section above, the inattentive type of ADHD had a higher prevalence (40.9\%) than the hyperactive-impulsive type (31.8\%) and the combined type (27.3\%). This is consistent with the findings of a study on university students in which a majority (56\%) of the respondents met criteria for the inattentive type while the remainder were equally distributed between the hyperactiveimpulsive and combined types (22\% each). ${ }^{13}$ However, a study on ADHD among university students in Eldoret, Kenya, found the hyperactive-impulsive type (40.5\%) to be more prevalent than the inattentive type (35.7\%) and combined type (23.8\%). ${ }^{16}$ These findings therefore demonstrate the need for further research in this area.

The main limitation in this study was that clinical examination was not used in this study to confirm the results of the ASRS screener or full symptom checklist. This may have resulted in a higher symptom prevalence rate than is expected, a property common to all screening instruments. However, feedback was provided to all participants indicating the score and its implications, including advice to seek a consultation with a mental health specialist where necessary. However, it is important to note that ASRS validation studies have yielded a high correlation with clinical diagnoses, raising the possibility that our findings have great clinical relevance as well. ${ }^{17,18}$

\section{Conclusion}

This study has demonstrated a high prevalence rate of selfreported $\mathrm{ADHD}$ symptoms among medical students in a low-income country. Apart from a decline of symptoms with increasing age, no other socio-demographic factors were shown to be significantly associated with ADHD symptom prevalence. The high prevalence rate demonstrates the importance of clinicians to have a high index of suspicion especially when handling students with academic and interpersonal difficulties. The study also demonstrates the need for more epidemiological studies on ADHD not only among students in schools and higher-level learning institutions but also in the general population.

\section{Acknowledgement}

The authors would like to thank all the medical students of Moi University Town campus who agreed to participate in this study.

\section{References}

1. American Psychiatric Association. Diagnostic and Statistical Manual of Mental Disorders, 4th ed, Text Revision. American Psychiatric Association, 2000.

2. Simon V, Czobor P, Balint S, Meszaros A, Bitter, I. Prevalence and correlates of adult attention-deficit hyperactivity disorder: meta-analysis. Br J Psychiatry 2009; 194:204-211.

3. DuPaul GJ, Schaughency EA, Weyandt LL, Tripp G, Kiesner J, Ota K, et al. Self-Report of ADHD Symptoms in University Students: Cross-Gender and Cross-National Prevalence. J Learn Disabil 2001; 34:370-379.

4. Montes LGA, García AOH, Ricardo-Garcell J. ADHD Prevalence in Adult Outpatients With Nonpsychotic Psychiatric Illnesses. J Atten Disord 2007; 11:150-156.

5. Fayyad J, de Graaf R, Kessler R, Alonso J, Angermeyer M, Demyttenaere $K$, et al. Cross-national prevalence and correlates of adult attentiondeficit hyperactivity disorder. Br J Psychiatry 2007; 190:402-409.

6. Polanczyk G, de Lima MS, Horta BL, Biederman J, Rohde LA. The Worldwide Prevalence of ADHD: A Systematic Review and Metaregression Analysis. Am J Psychiatry 2007; 164:942-948.

7. Kessler RC, Adler L, Barkley R, Biederman J, Conners CK Demler O, et al. The Prevalence and Correlates of Adult ADHD in the United States: Results From the National Comorbidity Survey Replication. Am J Psychiatry 2006; 163:716-723.

8. Hill JC, Schoener EP. Age-Dependent Decline of Attention Deficit Hyperactivity Disorder. Am J Psychiatry 1996; 153:1 143-1 146.

9. MCCarthy S, Asherson P, Coghill D, Hollis C, Murray M, Potts L, et al. Attention-deficit hyperactivity disorder: treatment discontinuation in adolescents and young adults.Br J Psychiatry 2009; 194:273-277.

10. Kolar D, Keller A, Golfinopoulos M, Cumyn L, Syer C, Hechtman L.Treatment of adults with attention-deficit/ hyperactivity disorder. Neuropsychiatric Disease and Treatment 2008; 4:389-403.

11. Weiss M, Murray C. Assessment and management of attention-deficit hyperactivity disorder in adults. CMAJ 2003; 168(6):715-22.

12. Faraone SV, Antshel KM. Diagnosing and treating attentiondeficit/hyperactivity disorder in adults. World Psychiatry 2008; 7:131-136.

13. Heiligenstein E, Conyers LM, Berns AR, Miller MA. Preliminary normative data on DSM-IV attention deficit hyperactivity disorder in college students. J Am Coll Health 1998; 46:185-8.

14. Weyandt LL, Linterman I, Rice JA. Reported Prevalence of Attentional Difficulties in a General Sample of College Students. Journal of Psychopathology and Behavioral Assessment 1995; 17(3):293-304.

15. Tuttle JP, Scheurich NE, Ranseen J. Prevalence of ADHD Diagnosis and Nonmedical Prescription Stimulant Use in Medical Students. Acad Psychiatry 2010; 34:220-223.

16. Atwoli L, Owiti P, Manguro G, Ndambuki D. Self-reported Attention Deficit and Hyperactivity Disorder symptoms among University Students in Eldoret, Kenya. East Afr Med J 2010; 87(5): 186-190

17. Kessler RC, Adler L, Ames M, Delmer O, Faraone S, Hiripi E, et al. The World Health Organization Adult ADHD Self-Report Scale (ASRS): A Short Screening Scale for Use in the General Population. Psychol Med 2005; 35:245-256

18. Kessler RC, Adler L, Gruber MJ, Sarawate CA, Spencer T, Van Brunt DL. Validity of the World Health Organization Adult ADHD Self-Report Scale (ASRS) Screener in a representative sample of health plan members. Int J Methods Psychiatr Res 2007; 16:52-65.

19. Shaffer D. Attention Deficit Hyperactivity Disorder in Adults. Am J Psychiatry 1994; 151:633-638. 